\title{
Etapas del proceso pedagógico
}

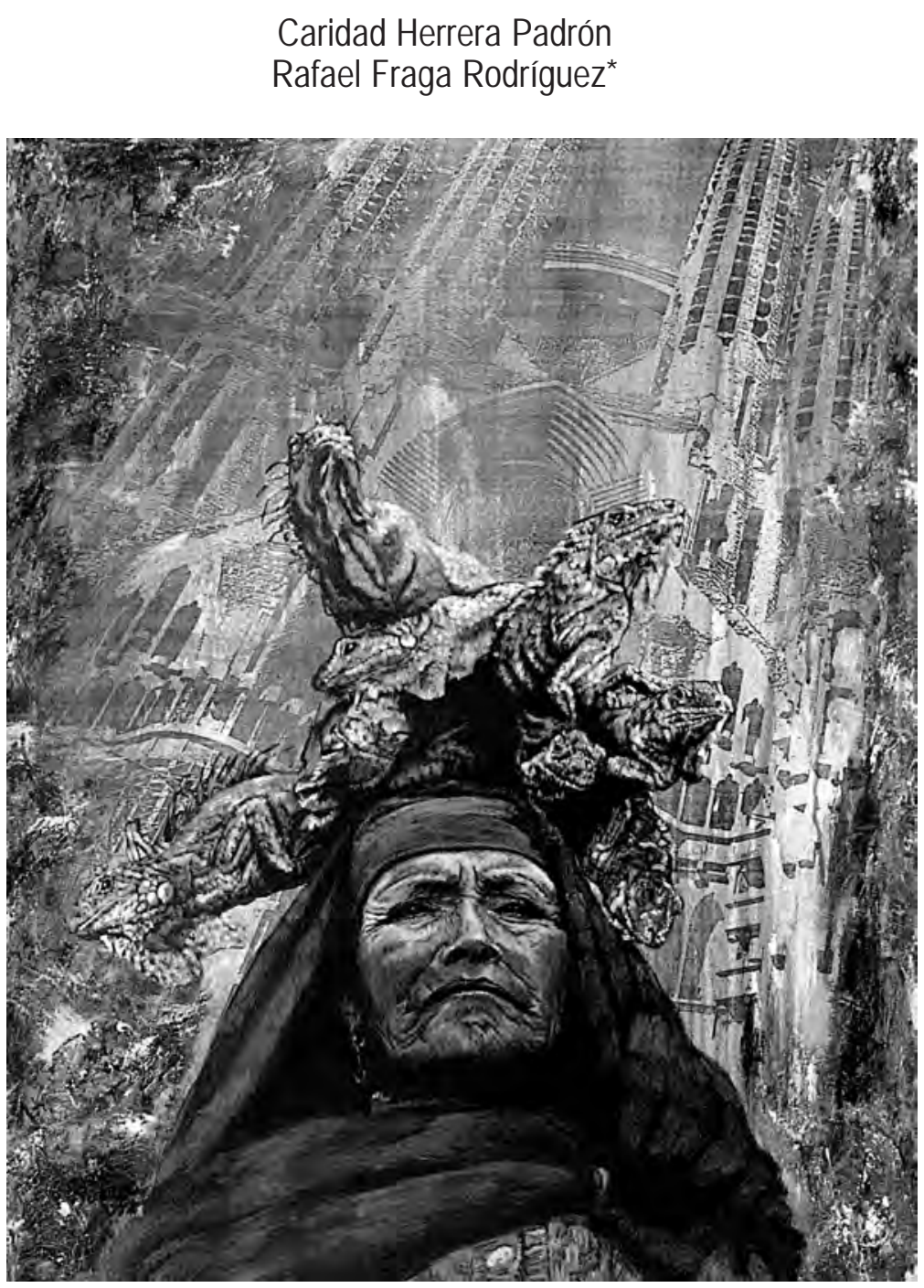

Chica cosmo. Óleo sobre tela. 2002

\footnotetext{
* Caridad Herrera Padrón: Magíster en Pedagogía Profesional. Profesora de Posgrados en universidades locales y del exterior; Rafael Fraga Rodríguez (PhD). Doctor en Ciencias Pedagógicas. Coordinador de Posgrados de la UNITA. Colaborador del CONESUP para los Diseños Curriculares, Director General de la Corporación CENIT. Profesor de Posgrados en universidades locales y del exterior.
} 
Como resultado de varias investigaciones realizadas en el campo de acción de la Didáctica y de la propia experiencia docente en diversos niveles educacionales, se puede presentar una propuesta de sucesión de etapas para gestionar el aprendizaje, que deben ser tomadas en consideración para alcanzar resultados satisfactorios en el proceso pedagógico.

Estas etapas son seis y se encuentran estrechamente interrelacionadas, su atención consciente en el desarrollo del proceso pedagógico permitirá el mejoramiento del aprendizaje en cualquier área del conocimiento.

A continuación se hace una caracterización de cada una de estas etapas.

\section{Etapa motivacional}

La etapa motivacional si bien se ubica para su análisis como la primera, esto de ningún modo quiere decir que será sólo en un primer momento del proceso; ésta se refiere al aseguramiento que el docente realiza conjuntamente con el estudiante de la predisposición positiva para la aproximación al nuevo contenido.

La motivación se refiere al interés desarrollado, a la expectativa positiva que el estudiante logra poner en juego por comprender de qué se trata el nuevo contenido. En ocasiones el estudiante ya posee esa motivación, en tal caso corresponde al docente después de auscultarla, realizar actividades para acrecentarla y mantenerla viva. En otros casos no viene dada esa predisposición y lógicamente se tendrán que buscar alternativas para que se presente. En esta última situación, generalmente tiene un efecto favorable la presentación y análisis de:

- historicidad del contenido y/o;

- paradojas relacionadas con el contenido y/o;

- problemas que no quedan dentro de la frontera de los contenidos dominados por los estudiantes y/o;

- $\quad$ exploración de aplicaciones prácticas y/o;

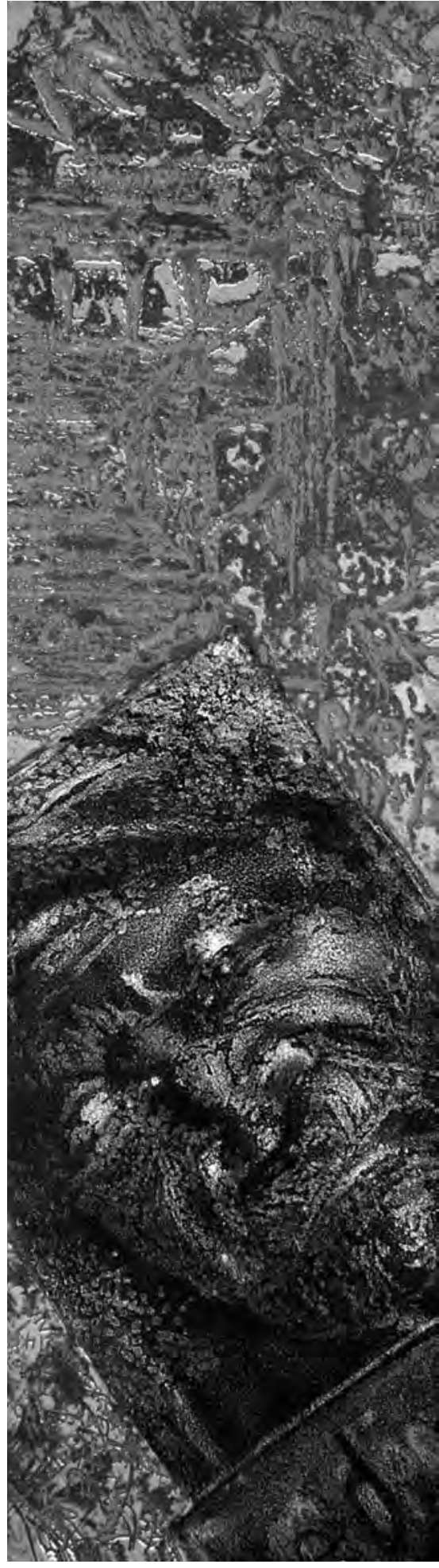

Esquina anónima. Roll up. 1996 
- hazañas relacionas con la temática, entre otras.

En todos los casos la alternativa que se utilice puede ser de diversa naturaleza procurando siempre que guarde una estrecha relación con el nuevo contenido y con las particularidades del grupo estudiantil. También es posible el uso de más de una de estas alternativas

Esta fase es muy conveniente para explorar, indirectamente, el nivel de partida o el dominio de contenidos previos necesarios para el nuevo aprendizaje.

Lamentablemente, muchos profesores piensan que puede ser una pérdida de tiempo; sin embargo la práctica demuestra que un estudiante motivado es capaz de avanzar con mayor

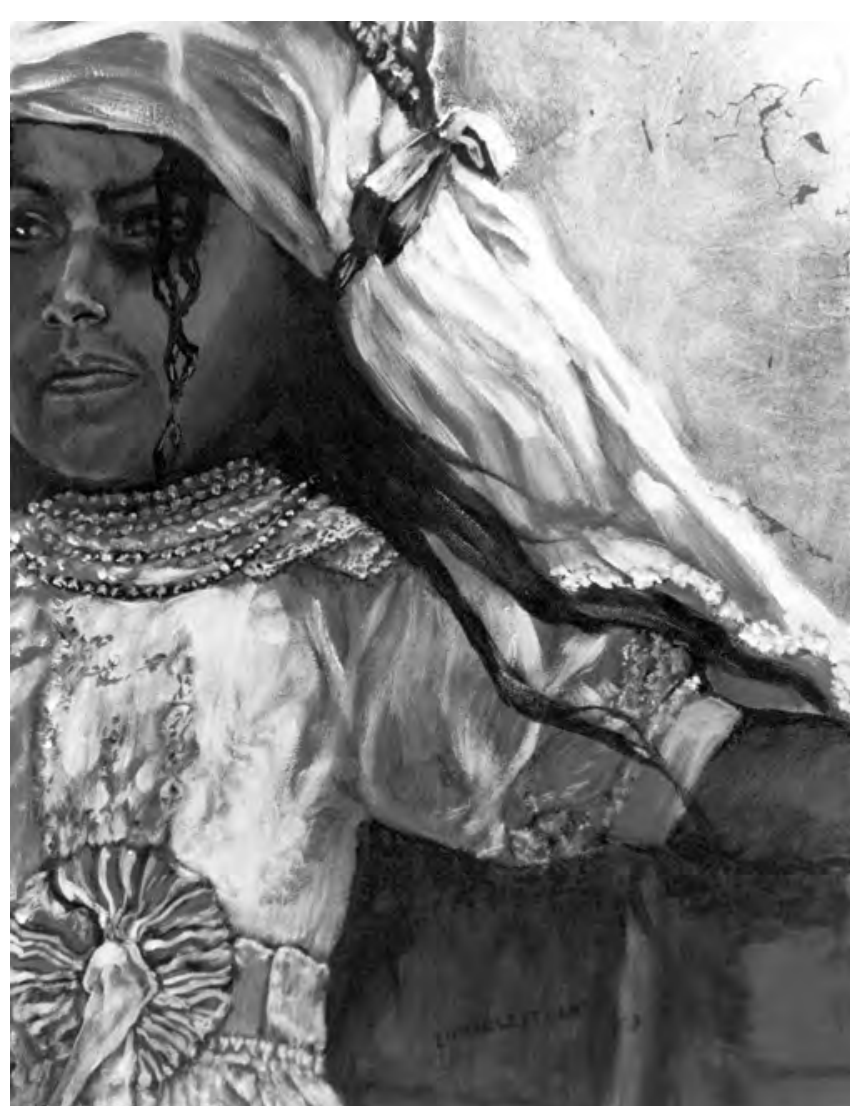

Niña ángel 1. Lámina de oro y acrílico sobre tela. 2003 independencia y dinamismo en la búsqueda del nuevo conocimiento, por lo que a la larga es una ganancia plena indiscutible.

\section{Apropiación del nuevo contenido}

Al iniciar esta etapa del proceso pedagógico, es necesario contar con estudiantes preparados para el esfuerzo de modificar su sistema de contenidos insertando los nuevos. No será posible que esta tarea de tan alta complejidad sea abordada sólo por el docente, por el contrario, éste debe preparar las condiciones para que tenga lugar esa incorporación.

En muchos casos es conveniente diseñar la aproximación al nuevo contenido utilizando métodos inductivo-deductivo, analítico-sintético o hipotético-demostrativo; aunque existen muchas otras alternativas.

Cada estudiante posee una representación mental del objeto de estudio, que no en todos los casos se corresponde con las del docente, ni con la de sus compañeros; por ello es necesario gestionar la apropiación con estrategias grupales e individuales, de modo que se manifieste la manera cómo se incorpora el nuevo contenido en relación a otros que ya se poseen.

Esta es una etapa de gran trascendencia para el estudiante y para el proceso en general, cada educando tendrá una manera propia de realizar la modificación de su estructura cognitiva y el docente debe controlar atentamente este proceso, sin pretender forzar la situación.

Por lo anteriormente explicado, es relevante realizar múltiples preguntas, mantener el diálogo, provocar que los estudiantes se pregunten entre ellos y realicen cuestionamientos al docente. Puede ayudar mucho la realización de diagramas, gráficos, organizadores gráficos; pero en todos los casos no se trata de que sean realizados por el docente sino que en la graficación el estudiante evidencie la estructura que está construyendo, estructurando, modificando. Así el do- 
cente estará en condiciones de prestar ayuda oportuna y necesaria.

En ocasiones un docente con apuro, no se detiene a observar a controlar a evaluar este proceso de tan alta complejidad; lo que traerá para el estudiante y para el proceso serias complicaciones.

Se reitera la necesidad de apreciar cómo se hace la construcción correspondiente, sin lo cual será baldío pasar a otra etapa.

En la etapa se hace referencia a la apropiación, considerando a ésta como el proceso de comprensión, de hacer suyo el nuevo contenido; vale aclarar que este es un proceso y no un estadio, es evolutivo, cada vez se van alcanzando niveles superiores de dominio; este no es un camino con final definido, es por aproximaciones sucesivas. Por esta razón, el educador debe ser paciente, persistente y comprensivo tanto en la dirección del proceso como en la evaluación de los avances en la apropiación.

\section{Fijación del nuevo contenido}

Una vez alcanzada la apropiación del nuevo contenido, es necesario garantizar que se fije en el estudiante, esto se logra mediante la elaboración de tareas de diferente naturaleza. Algunas tendrán una base de interpretación, otras estarán orientadas a la utilización de algoritmos que ayuden en la automatización de determinadas acciones y fijación de procesos, otras permitirán que el estudiante ponga a prueba el dominio teórico y práctico del contenido de estudio y, por supuesto, habrá tareas donde se ponga de manifiesto la creatividad del estudiante.

Esta etapa pudiera denominarse como entrenamiento en la utilización del nuevo contenido, la cual es de alta trascendencia si se quieren alcanzar resultados relevantes en el aprendizaje.

No basta con la apropiación, comprensión y hasta reproducción del contenido, es imprescindible alcanzar una buena fijación, lo que garantizará solidez a futuro.
La realización de ejercicios y problemas deben cubrir la más amplia gama de posibilidades para que en lo ulterior, el estudiante pueda recurrir a estos contenidos cuando sea necesario.

\section{Etapa de aplicación del contenido}

Esta fase corresponde a la puesta en práctica de los nuevos contenidos en diversidad de situaciones y contextos, para alcanzar la distinción entre aspectos invariantes y las variantes que se pueden presentar con el nuevo contenido fijado.

La realización de problemas y el enfrentamiento de nuevas situaciones utilizando las po-

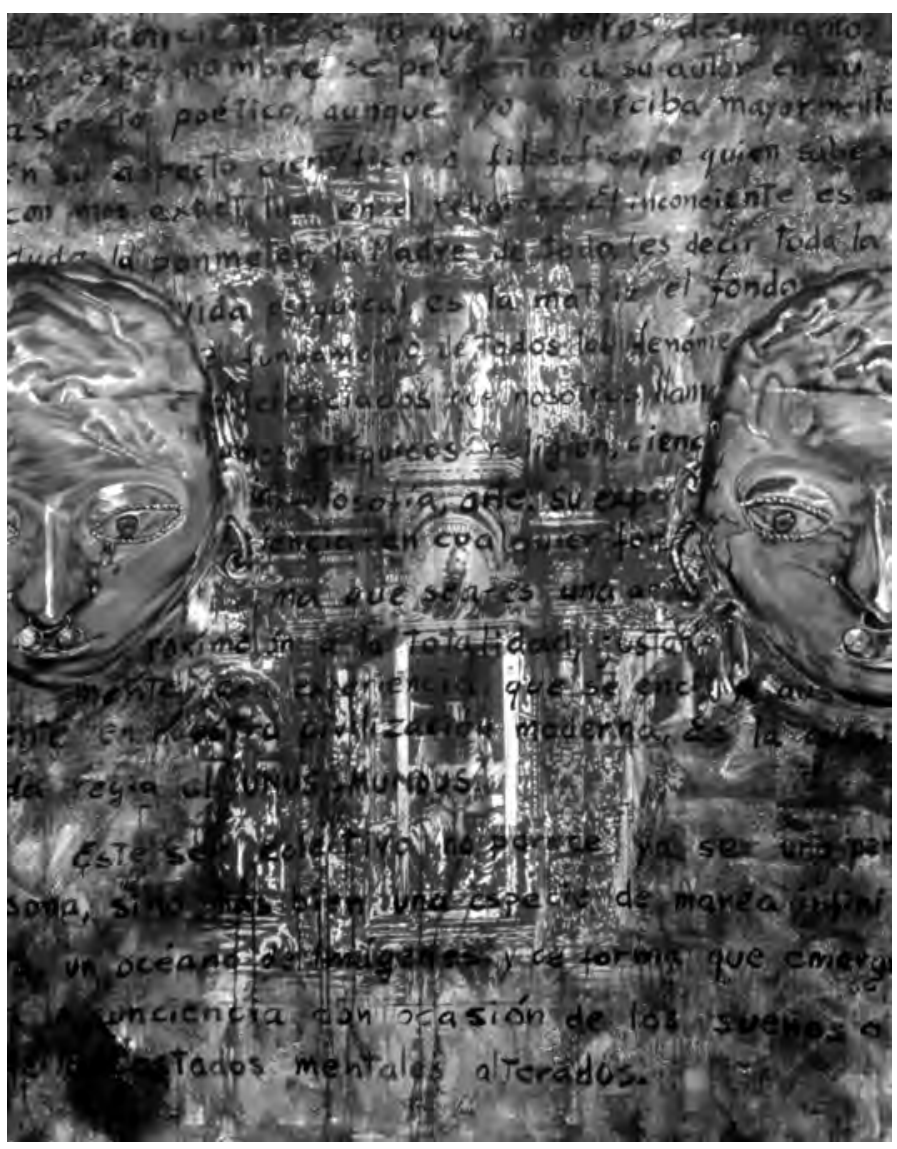

Inconsciente colectivo. Estampación y acrílico sobre tela. 2003 
tencialidades alcanzadas, contribuye al proceso de aplicación del contenido.

La etapa de aplicación del contenido pone a prueba las potencialidades individuales respecto a nuevas exigencias unas veces formuladas por el docente y otras por los mismos estudiantes.

Del mismo modo y como parte de la preparación del estudiante será necesario pedirles que determinen y formulen problemas relacionados con el contenido objeto de estudio.

Además, la etapa de aplicación permitirá utilizar ajustes y extrapolaciones necesarios para asegurar el pleno dominio.

\section{Etapa de profundización del contenido}

Subsiguiente a la etapa de aplicación corresponde otra que está íntimamente relacionada con el dominio profundo del contenido. La profundización en el contenido no se refiere a un determinado nivel de apropiación (familiarización, reproducción, producción, aplicación, creación...), sino a la riqueza que se tiene en su dominio.

La profundización es el periodo que trata de cómo el estudiante enriquece su comprensión por medio de la búsqueda, la discusión y análisis de hallazgos científicos o tecnológicos relacionados con el objeto de estudio. Por ello, la orientación del estudio independiente es necesaria, así como el empleo de formas organizativas como el seminario y el taller, donde el estudiante exponga nuevas ideas, interpretaciones, descubrimientos relacionados al tema en estudio.

En general esta etapa se conforma por la intervención individual y la construcción colectiva en el grupo de aprendizajes, el papel fundamental del profesor es de animador, organizador, moderador y guía experimentador.

Cuando se ha profundizado en el tema, se aprecian nuevas interconexiones que aseguran el aprendizaje.

\section{Etapa de sistematización del contenido}

Independientemente que se presenta como una etapa necesaria dentro del proceso pedagógico, no se refiere a un momento específico dentro de la clase o del tema, su comportamiento es inmanente a todo el proceso de enseñanzaaprendizaje.

Pudiera decirse que se comporta más como un principio, que como un periodo del proceso pedagógico.

Consiste en que el estudiante sea consciente de cómo él comprende, aprecia, interrelaciona los diferentes contenidos que son objeto de aprendizaje. ¿Cómo se relaciona el nuevo contenido con el resto de contenidos, bien sean de una o de varias disciplinas? ¿Cuáles son las relaciones de jerarquía y coordinación que va descubriendo? ¿Cómo es utilizado en la práctica? En fin, la sistematización logrará que el nuevo contenido quede sujeto dentro de una trama de relaciones que el estudiante ha ido construyendo a lo largo de su experiencia vital, y de esta manera se constituye en un cuerpo estructurado, complejo de relaciones que reflejan el aprendizaje de cada persona.

La sistematización está siempre presente en el proceso pedagógico, pero en ocasiones es espontánea y como tal no se gestiona ni se evalúa.

Cuando se brindan las pautas necesarias para que el estudiante conforme su propio sistema de contenidos, se puede apreciar la fortaleza, la seguridad que el estudiante demuestra en diferentes situaciones.

Tener en cuenta estas etapas dentro del proceso pedagógico, lleva implícito trabajar en función de la sistematización en el mismo, puesto que cada una de ellas tiene su propia función dentro del proceso de aprendizaje de los nuevos contenidos, además de estar interrelacionadas entre sí.

No será posible la aplicación si antes no se fija el contenido y éste no podrá tener efecto si previamente no se ha apropiado el estudiante del mismo. 


\section{Palabras clave}

\section{- Contenido}

Sistema de conocimientos habilidades, cualidades de la personalidad, destrezas, competencias y otros elementos que conforman la cultura, por cuanto el contenido objeto de aprendizaje es una parte de la cultura, que se considera necesario tener en cuenta en el currículo de estudio de un nivel educativo determinado.

\section{- Proceso pedagógico}

Proceso que se da en las instituciones docentes, con el fin de que los estudiantes incorporen a sus experiencias de aprendizaje los contenidos que portan los diseños curriculares. Algunos llaman a éste, proceso docente educativo o proceso de enseñanza aprendizaje.

\section{- Aprendizaje}

Proceso y resultado, mediante el cual, el sujeto va incorporando nuevas experiencias (conocimientos, habilidades, capacidades y cualidades) que le permiten actuar ante las diversas situaciones que pueden presentarse.

El tema acordado constituye un aspecto importante dentro del contenido de la Didáctica, las etapas brindan una descripción de los momentos por los que deben transitar los estudiantes para lograr una apropiación sólida del contenido del aprendizaje.

Las etapas son segmentos del proceso, en ellas están presentes todos los elementos constitutivos del proceso pedagógico.

\section{Bibliografía}

- FRAGA RODRIGUEZ, Rafael: La Sistematización de la Trigonometría en el Nivel Medio Superior en Relación con las Exigencias del Nivel Superior. Tesis de
Grado Científico. Ciudad de la Habana. 1992

- HERRERA PADRÓN, Caridad y Rafael Fraga Rodríguez: Didáctica de la Educación Superior. Material de Apoyo del Módulo de Didáctica de la Maestría en Educación, Quito: UNITA. 2008

- HERNÁNDEZ FERNÁNDEZ, Ana M. y Rafael Fraga Rodríguez: Hacia una Eficiencia Educativa. Quito-Ecuador. 1994

- TALÍZINA, Nina: Conferencias sobre los fundamentos de la Enseñanza en la Educación Superior. La Habana: CEPES. 1984

- VIDAL, J.A. y Grupo Editorial: Enciclopedia General de la Educación. En 3 Tomos. Barcelona: Editorial Océano. 1999

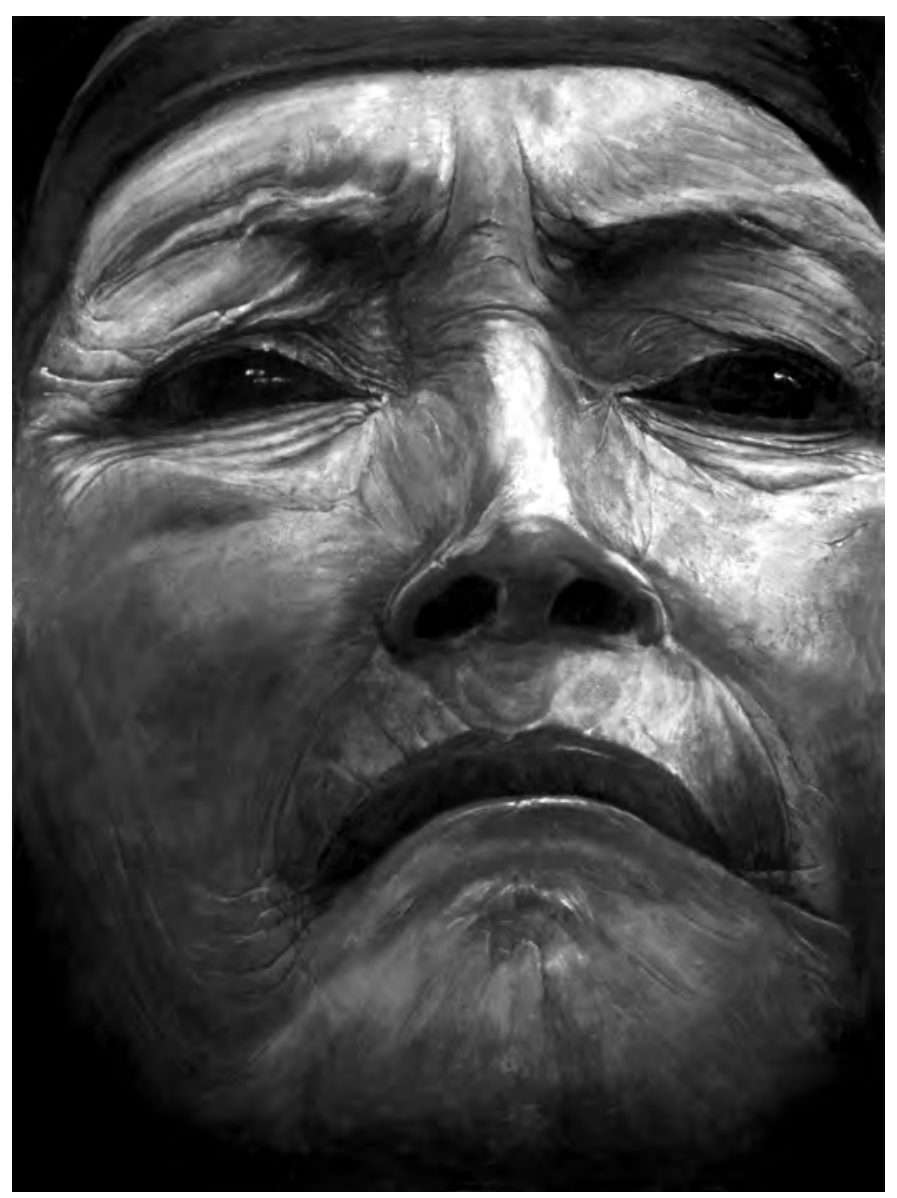

Quilago (32 módulos). Acrílico sobre tela. 2004 